\title{
Improved Method for Differentiation of Synthetic and Natural Endogenous Anabolic Steroids using Gas Chromatography Isotope Ratio Mass Spectrometry (GC/C/IRMS) followed by Two-Fold High Performance Liquid Chromatography (HPLC) Cleanup Method: A Perspective
}

\author{
Abhinav Shrivastava1, Shila Jain', Puran Lal Sahu1, Sangeeta Shukla², Kapendra Sahu* \\ ${ }^{1}$ National Dope Testing Laboratory, Ministry of Youth Affairs and Sports, JLN Stadium Complex, Government of India, New Delhi, INDIA. \\ ${ }^{2}$ School of Studies in Zoology, Gwalior, Madhya Pradesh, INDIA.
}

\begin{abstract}
Background: Androgenic Anabolic Steroids (AAS) are also synthetic derivatives of testosterone, modified to improve its anabolic actions. The misuse of AAS is of particular concern in sports and society. Gas chromatography-mass spectrometry had some limitations and allows identification and characterization of steroids and their metabolites in the urine but may not be able to distinguish between pharmaceutical (Exogenous) and endogenous origin. Thus, it is of great importance to discriminate endogenous steroids such as testosterone or testosterone prohormones from their chemically identical synthetic copies. The abuse of Androgenic Anabolic Steroids (AAS) by sports person is banned by World Anti-doping Agency (WADA) as per the WADA Prohibited list 2019. Methodology: The gas chromatography-combustion/isotope ratio mass spectrometry (GC/C/IRMS) technique differentiates between natural and synthetic endogenous steroids by comparing compounds specific ${ }^{13} \mathrm{C} /{ }^{12} \mathrm{C}$ ratio. However, the analytes have to be efficiently isolated and purified prior to GC/C/IRMS analysis. Results and Discussion: HPLC Cleanup method prior to analysis by GC-C/IRMS needs to be developed and validated for discriminating the origin of anabolic androgenic steroids. These methods involves the solid-phase extraction, enzymatic hydrolysis with $\beta$-glucuronidase, HPLCfractionation for the cleanup and analysis by GC-C/IRMS. The difference $\left(\Delta^{13} \mathrm{C}\right)$ of urinary $\delta^{13} \mathrm{C}$ values between synthetic analogues and Endogenous Reference Compounds (ERC) by GC-C/IRMS would be used to elucidate the origin of steroids. The present perspective gives an overview of the use of anabolic-androgenic steroids in sport and methods used in anti-doping laboratories for their detection in urine, with special emphasis on $\mathrm{GC}-\mathrm{C}$ / IRMS technique after two-fold HPLC cleanup.
\end{abstract}

Key words: Endogenous Steroids, Exogenous Steroids, HPLC Cleanup, Doping, GC/C/ IRMS.

\section{INTRODUCTION}

The misuse of synthetic endogenous steroid copies is one of the most important issues in sports. Athletes may abuse steroids or manipulate metabolic pathways in an attempt to increase concentrations of biologically active steroids with the intent of enhancing athletic performance through increased muscle mass and more rapid recovery from injury or intense training. ${ }^{1}$ Therefore, the administration of steroids has been strictly prohibited by World AntiDoping Agency (WADA) and it is necessary to discriminate endogenous steroids from their chemically identical synthetic copies
Submission Date: 03-05-2019; Revision Date: 04-09-2019; Accepted Date: 30-09-2019

DOI: 10.5530/ijper.53.4s.139 Correspondence: Dr. Kapendra Sahu, M.Pharm, Ph.D, National Dope Testing Laboratory, Ministry of Youth Affairs and Sports (MYAS), Government of India, Lodhi Road-110003, New Delhi, INDIA.

Phone: +91 011-24368850 E-mail: kapendra@gmail. com

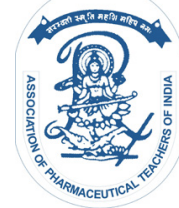

www.ijper.org 
for elucidating the origin of steroids. ${ }^{2}$ Traditionally, the GC-MS method has been used for the detection of steroids. ${ }^{3}$ However, this method has limitation on distinguishing endogenous steroids such as testosterone or testosterone prohormones from the chemically identical endogenous synthetic copies. In steroids, differences in ${ }^{13} \mathrm{C}$ content arise because synthetic steroids are derived from plant sterols such as stigmasterol and sitosterol obtained from C-3 plants that constitute about $90 \%$ of all plants. ${ }^{4}$ They usually exhibit depleted ${ }^{13} \mathrm{C} /{ }^{12} \mathrm{C}$ ratio $\left(\delta^{13} \mathrm{C}\right)$ values in range $-25.9 \%$ to $-35.6 \%$ in comparison with the values for endogenous steroids. ${ }^{5}$ Based on $13 \mathrm{C}$ isotopic differences, the measurement of $\delta 13 \mathrm{C}$ of steroids is highly regarded to elucidate the origin of steroids and the use of gas chromatography combustion isotope ratio mass spectrometry (GC-C/IRMS) has proven to be the unique analytical technique of choice in this field. ${ }^{6-10}$ Thus, the analysis and difference $\left(\Delta^{13} \mathrm{C}\right)$ of urinary $\delta^{13} \mathrm{C}$ values between synthetic analogues and Endogenous Reference Compounds (ERC) such as 11-keto-etiocholanolone, 11ß-OH-androsterone and pregnanediol allows endogenous steroids to be distinguished from their synthetic analogues in the urine and provides significant information that they have not administrated synthetic analogues of endogenous steroids. ${ }^{11-13}$ The detection of endogenous steroids (found naturally in the body) is a challenge faced by Doping Control Laboratories across the world. In the present study, we validated a comprehensive GC-C/IRMS method combined with HPLC cleanup for the discrimination of endogenous steroids and successfully applied to the urine samples of endogenous synthetic steroids abusers especially in the cases when confirmation for the low concentration metabolites was to be made. Analytes have to be efficiently isolated and purified before GC/C/IRMS analytes in order to avoid any co-elution of compounds. Therefore, extensive sample preparation followed by HPLC cleanup was employed. Moreover, it was reviewed in literature that HPLC Cleanup was accurate and reproducible enough to be successfully applied to the test of urine sample from suspected anabolic steroid abusers.

\section{Methods for Urinary Steroid Analysis}

\section{Reagents and Chemicals}

Reference standards of endogenous steroids and deuterated internal standards were procured from Sigma-Aldrich, USA, or National Measurement Institute, Australia. 11-keto-etiocholanolone (11-keto) was obtained from German laboratory. $\mathrm{C}_{18}$ sample preparation cartridges were procured from RFCL Ltd and $3 \mathrm{M}$ Empore, Varion. $\beta$-glucuronidase enzyme from $E$. coli was from Roche Diagnostics, USA. Methanol $(\mathrm{MeOH})$ (HPLC-grade) and acetonitrile (ACN) (HPLC-grade) was purchased from Merck (USA). HPLC grade water was prepared using a Milli-Q purification system (Millipore, Bedford, MA).

\section{Urine steroid profile determination}

Routine screening procedure consisting of solid phase clean up, enzymatic hydrolysis, solvent extraction and derivatization, followed GC-MSD analysis was implemented to determine the concentration of Androgenic Anabolic Steroids (AAS), as described previously. ${ }^{14-15}$

Sufficient clean up of all analytes was achieved by a two fold HPLC fractionation comprising first of underivatized and then acetylated steroids. The clean up was performed on Waters Alliance 2695 separation module with automated fraction collector WFC-3, equipped with Merck analytical column (LiChroCART 250 x 4 mm i.d., $5 \mu \mathrm{m}$ particle size) and guard column (LiChroCART 25 $\mathrm{X} 4 \mathrm{~mm}$ i.d., $5 \mu \mathrm{m}$ particle size). For the first run, a linear gradient increasing from acetonitrile: water (30:70) to $100 \%$ acetonitrile in $25 \mathrm{~min}$ was used. After $5 \mathrm{~min}$ at $100 \%$ acetonitrile, the column was re-equilibrated for $5 \mathrm{~min} ; 50 \mu \mathrm{L}$ injection volume and flow rate of $1 \mathrm{ml} /$ min. Before each batch of samples, a standard comprising of $100 \mu \mathrm{g} / \mathrm{ml}$ of 11-keto-etiocholanone (11-keto), Testosterone (T), Epi-testosterone (EpiT), Androsterone (A), Etiocholanone (E), Pregnanediol (PD) and $\beta$-Estradiol-3, 17 diacetate (EST) were injected twice to determine the retention times for fraction collection. HPLC chromatograms of the first clean up along with fraction collection window are shown in Figure 1. Fraction I, IV and V were acetylated and injected onto GC-C-IRMS. Fraction II and III from the first run was acetylated and purified again to achieve sufficient clean up.

For second HPLC clean-up, a different gradient was used. From 70/30 acetonitrile/water, a linear increase to $100 \%$ acetonitrile was accomplished in $33 \mathrm{~min}$ and

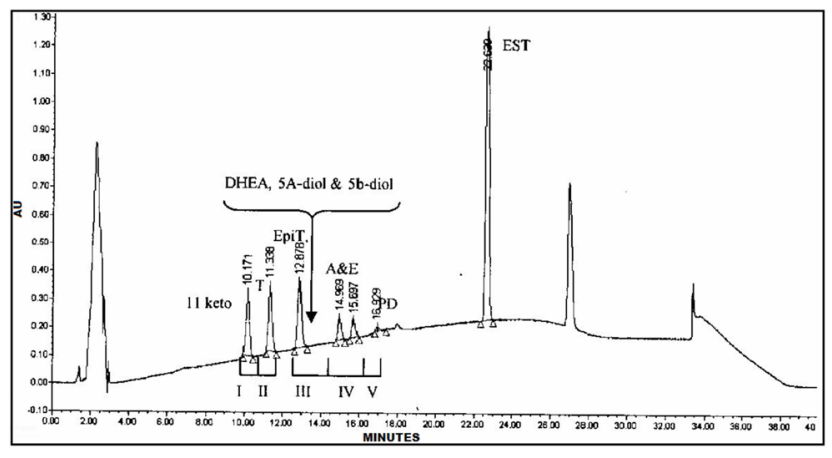

Figure 1: HPLC Chromatogram of standard injection for $1^{\text {st }}$ Fraction Collection at wavelength of $192 \mathrm{~nm}$ with fraction collection. 
maintained for 5 min. subsequently the column was reequilibrated for $5 \mathrm{~min}$. A standard mixture containing TAc, RSTDAc, EST, 5a ( $5 \alpha$-androstane- $3 \alpha, 17 \beta$-diol) Ac, $5 b$ ( $5 \beta$-androstane- $3 \alpha, 17 \beta$-diol) Ac, DHEA (dehydroepiandrosterone) Ac, EpiT Ac $(100 \mathrm{ug} / \mathrm{ml})$ in 70:30 acetonitrile: water in HPLC to know the RTs for fraction collection. 17 $\beta$-estradiol-diacetate (RSTD) was used as reference standard for HPLC to control the acetylation step. Figure 2 shows the HPLC chromatogram of second clean up along with fraction collection windows.

\section{Determination of Carbon Isotope Ratio (CIR)}

Analytes have to be efficiently isolated and purified before GC/C/IRMS analytes in order to avoid any coelution of compounds. Therefore, extensive sample preparation followed by HPLC clean up was employed. The samples were tested on GC/C/IRMS and ${ }^{13} \mathrm{C} /{ }^{12} \mathrm{C}$ ratios of endogenous steroids (Androsterone, Etiocholanolone, 5a and 5b-androstan-3a,17b diols, Testosterone DHEA and Epitestosterone and ISTD-RSTD) along with ERCs (11 Keto-etiocholanolone, Pregnanediol and ISTD-RSTD) were measured. The QC Negative and QC Positive used were Drug Free Urine from a healthy male volunteer and pooled excretion study samples (1-61 hr) from healthy male volunteer by administration of Oral Testosterone undecanoate (40 mg).

\section{GC/MS Identification}

In order to detect and identify co-elutions and to ensure the absence of any disturbing matrix components in all fractions it was necessary to scan all samples on a GC-MS system using chromatographic conditions equivalent to the IRMS set-up during method development. For this purpose, GC Agilent 6890 coupled to a mass selective detector was used. The MSD data was acquired in scan mode from $\mathrm{m} / \mathrm{z}, 40$ to 400 and mass spectral data was compared to standards.

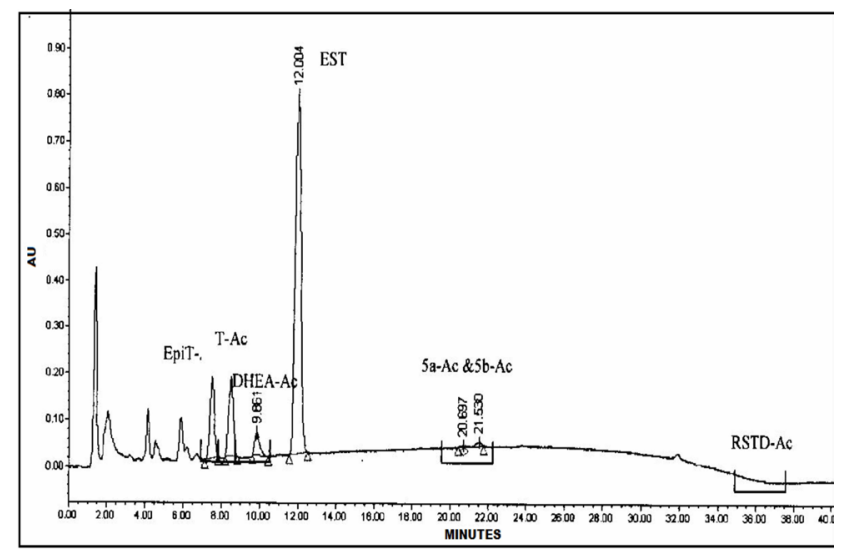

Figure 2: HPLC Chromatogram of standard injection for second clean up at $192 \mathrm{~nm}$ with Fraction collection.

\section{RESULTS AND DISCUSSION}

The endogenous steroids which are excreted in low concentration show improved separation by HPLC Cleanup procedure as it removes all the interfering coeluting compounds and thus proves to be more useful for the confirmation of suspicious samples in routine testing. The different purified fractions along with their $\mathrm{R}_{\mathrm{T}}$ is shown in Table 1 .

The aim of this study to develop and validate the GC/C/ IRMS method for the discrimination of endogenous steroids and demonstrate that the present method is useful for the identification of the anabolic androgenic steroids abusers was achieved. An initial effort for optimizing the sample preparation was made to achieve more complete enzymatic hydrolysis to convert glucuronide conjugated metabolites of the target endogenous steroids into their free form and clean isolation of the hydrolyzed target analytes to minimize the endogenous interferences. For these purposes, the solid-phase extraction was employed prior to the enzymatic hydrolysis and HPLC-cleanup of the hydrolyzed sample. Seven fractions were collected for the isolation of 10 endogenous steroids using the HPLC-cleanup condition described in Table 2. After dryness and reconstitution of fractions, the resulting samples were analyzed by GC-C/ IRMS with derivatization step. Figure 3-11 illustrates representative chromatograms of each fraction obtained from

\begin{tabular}{|c|c|c|}
\hline $\begin{array}{c}\text { Table 1: Fraction collection program for pure form of } \\
\text { urinary steroids purified by HPLC }\end{array} \mathbf{1}^{\text {st }}$ & Cleanup. \\
\hline $\begin{array}{c}\text { HPLC } \\
\text { fraction No. }\end{array}$ & Compound Names & $\begin{array}{c}\text { Retention } \\
\text { times (min.) }\end{array}$ \\
\hline F-1 & 11 Keto Etiocholanolone & $9.4-10.4$ \\
\hline F-2 & $\begin{array}{c}\text { Testosterone } \\
\text { Epitestosterone, 5a-ADiol, } \\
\text { 5b-ADiol, DHEA }\end{array}$ & $11-12$ \\
\hline F-3 & $\begin{array}{c}\text { Androsterone and } \\
\text { Etiocholanonone }\end{array}$ & $14.4-14.2$ \\
\hline F-4 & Pregnanediol & $16.3-17.4$ \\
\hline F-5 & & \\
\hline
\end{tabular}

Table 2: Fraction collection program for acetate urinary steroids purified by HPLC $2^{\text {nd }}$ Cleanup.

\begin{tabular}{|c|c|c|}
\hline $\begin{array}{c}\text { HPLC fraction } \\
\text { No. }\end{array}$ & Compound Names & $\begin{array}{c}\text { Retention } \\
\text { times (min.) }\end{array}$ \\
\hline F-2 & Testoaterone -Acetate & $8.3-9.3$ \\
\hline F-3-1 & Epitestosterone-Acetate & $7.2-8.3$ \\
\hline F-3-2 & $\begin{array}{c}\text { Dehydroepiandrosterone- } \\
\text { Acetate }\end{array}$ & $9.1-10.1$ \\
\hline F-3-3 & $\begin{array}{c}\text { 5a-ADiol-Diacetate, } \\
\text { 5b-ADiol-Diacetate }\end{array}$ & $20-22$ \\
\hline
\end{tabular}




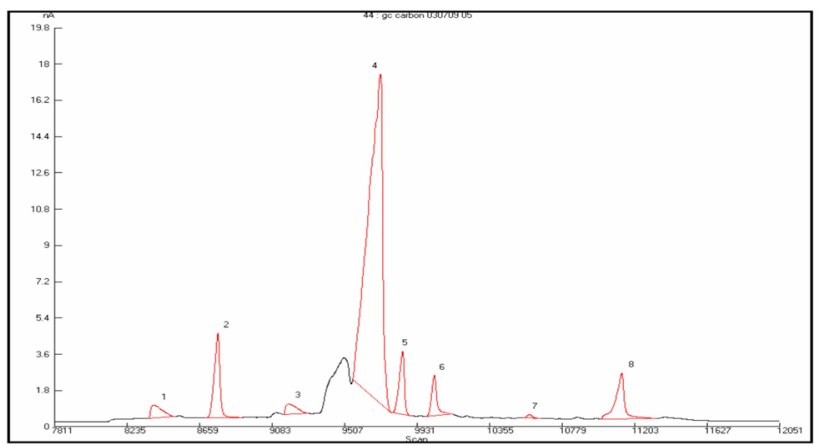

Figure 3: IRMS chromatogram of a sample injected previously without HPLC cleanup showing problem of co elution and absence of various compounds/peaks of interest.

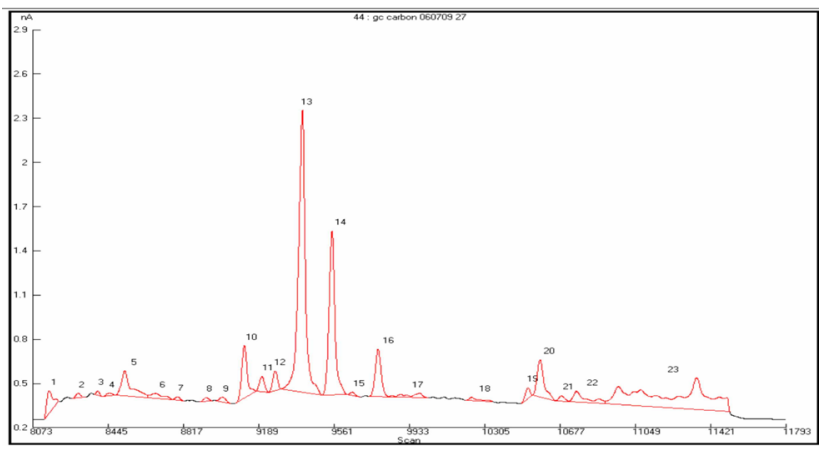

Figure 4: IRMS chromatogram of a sample injected previously without HPLC cleanup showing problem of uneven baseline and various interfering peaks.

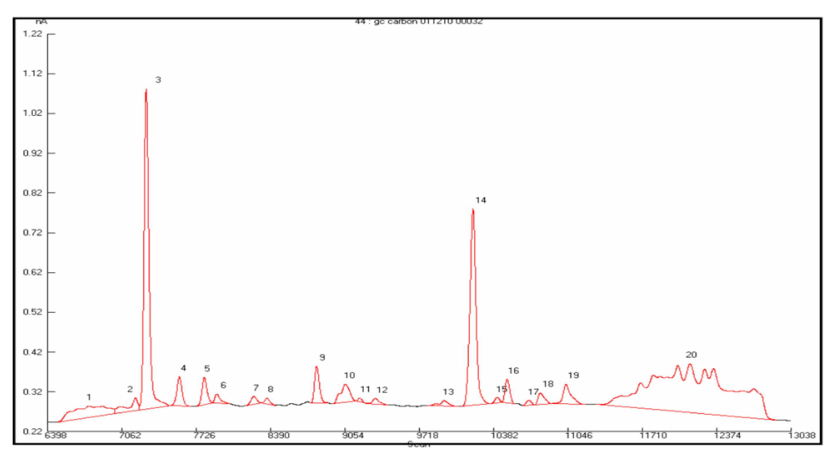

Figure 5: Fraction 1 from first fractionation showing concentrated and separate peaks of RSTD (3) and 11-Keto-etio (14).

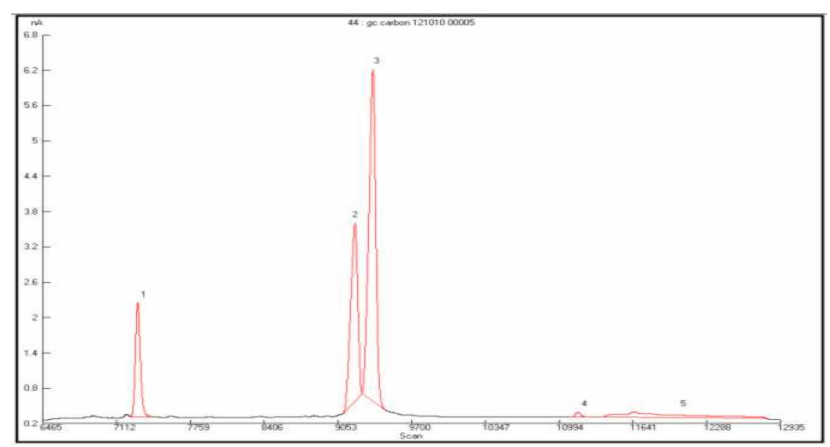

Figure 6: Fraction 4 from First Fractionation showing concentrated separate peaks of Androsterone (3) and Etiocholanolone (2) along with RSTD (1).

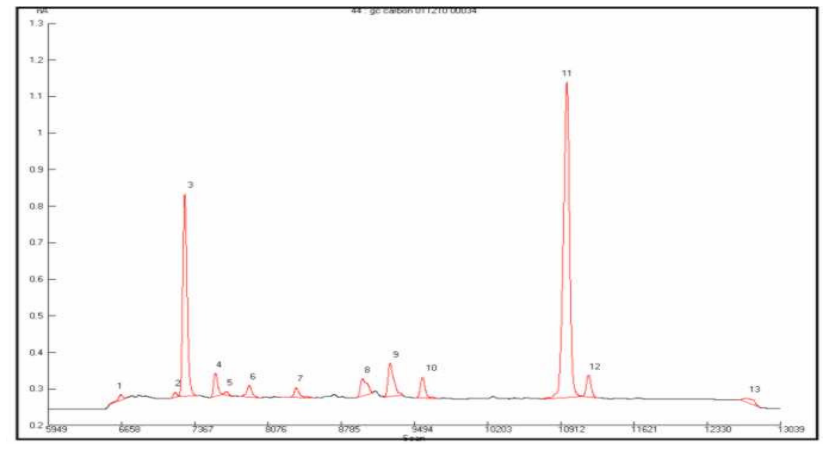

Figure 7: Fraction 5 from First Fractionation showing concentrated separate peaks of Pregnandiol (11) along with RSTD

(3).

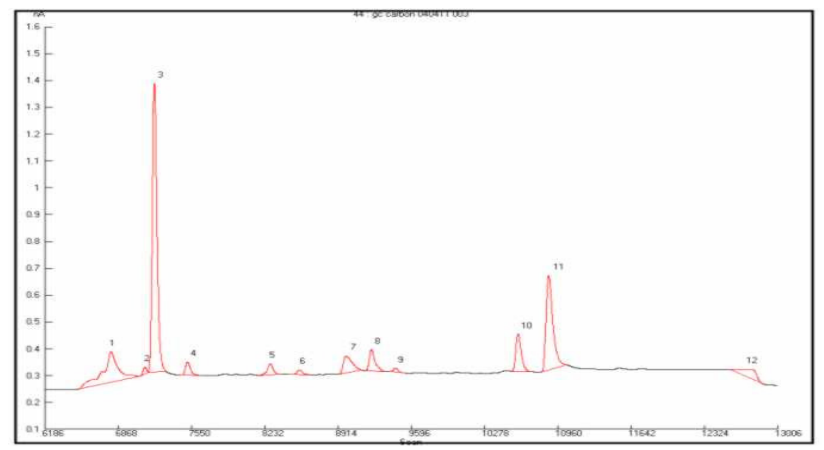

Figure 8: Pooled fraction 2 after second fractionation showing concentrated separate peaks of Testosterone (11) along with RSTD (3).

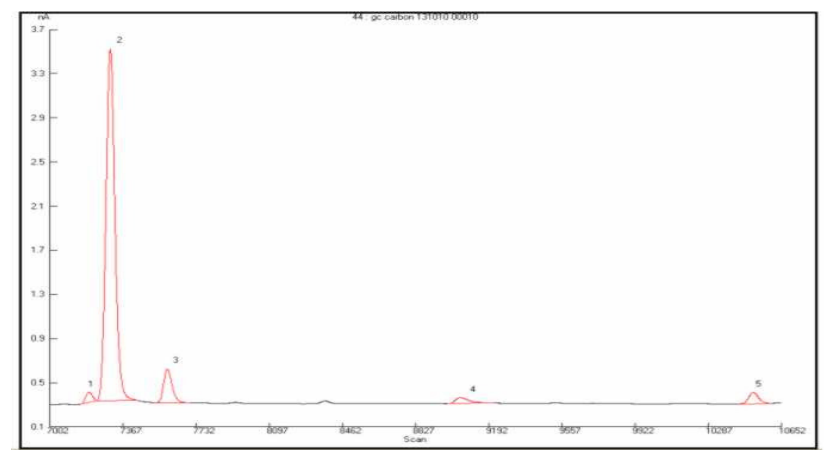

Figure 9: $1^{\text {st }}$ fraction of Fraction 3 after second fractionation showing concentrated separate peaks of Epitestosterone (5) along with RSTD (2).

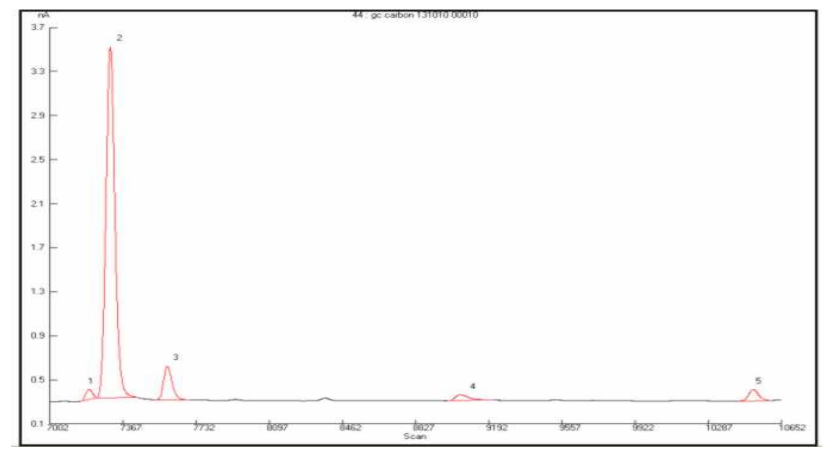

Figure 10: $2^{\text {nd }}$ fraction of Fraction 3 after second fractionation showing concentrated separate peaks of DHEA (5) along with RSTD (2). 


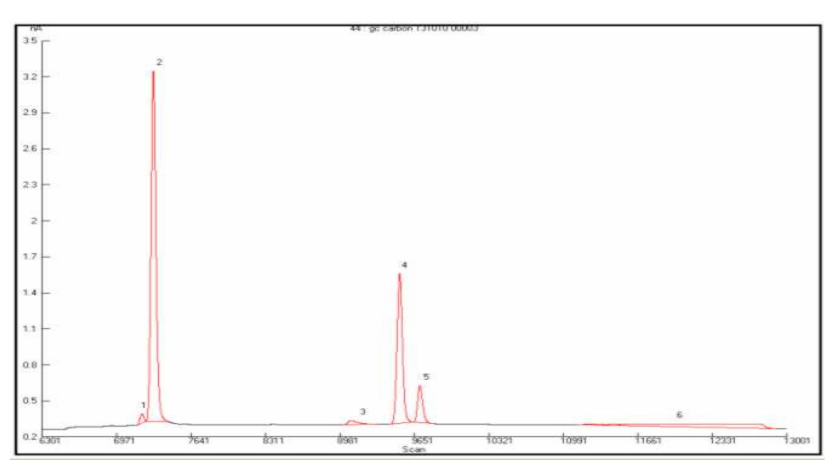

Figure 11: $3^{\text {rd }}$ fraction of Fraction 3 after second fractionation showing concentrated separate peaks of 5b-ADiol-acetate (4), 5a-ADiol-acetate (5) along with RSTD (2).

the urine samples. No significant interfering peak of urine matrix was observed at the retention time of all steroids and the retention times of target analytes were consistent with those of standards. Identification of target urinary steroids was confirmed by comparison with mass spectra of standards obtained from GC-MS analysis. ${ }^{16,17}$ The present method for the discrimination of endogenous steroids was validated. The urinary $\delta^{13} \mathrm{C}$ values for 10 endogenous steroids were obtained and intra- and inter-day precision and isotopic fractionation were evaluated to be reproducible in the measurement of urinary $\delta^{13} \mathrm{C}$ values. Sample preparation steps such as the SPE and HPLC-cleanup may seriously give rise to the isotopic fractionation phenomenon affecting the accuracy of IRMS analyses. To determine potential ${ }^{13} \mathrm{C}$ isotopic fractionation of the steroids during the sample preparation, the isotopic fractionation test was conducted. When comparing with $\delta^{13} \mathrm{C}$ values of standards, $\delta^{13} \mathrm{C}$ values of the analytes from urine samples were consistent with those of standards (RSTD) suggesting that no significant isotope fractionation effect was observed.

\section{CONCLUSION}

In continuation of earlier reviews, the present study summarizes the various approaches undertaken in expanding knowledge and improving method for GCC-IRMS in with regard to human doping controls. GC-C/IRMS method combined with HPLC-cleanup has been developed and validated for the discrimination of endogenous steroids in human urine. The method is much better than the previous method using Solid Phase Extraction (SPE) only. This method was successfully applied to analysis of the urine samples from the suspected synthetic testosterone abusers in CWG 2010 and SYOG 2010 in presence of experts from national and international laboratories. As results, the present method is useful to elucidate the origin of the endog- enous steroids even with metabolites which are very low in concentration.

\section{ACKNOWLEDGEMENT}

The author wishes to acknowledge the Ministry of Youth Affairs and Sports, Govt. of India for their continuous support.

\section{CONFLICT OF INTEREST}

The authors declare no conflict of interest.

\section{ABBREVIATIONS}

AAS: Androgenic Anabolic Steroids; GC/C/IRMS: Gas chromatography-combustion/isotope ratio mass spectrometry; WADA: World Anti-Doping Agency; ERC: Endogenous Reference Compounds; HPLC: High Performance Liquid Chromatography; GC-MSD: Gas Chromatography-Mass Selective Detector; SPE: Solid Phase Extraction; CIR: Carbon Isotope Ratio; RSTD: Reference Standard; $\mathbf{R}_{\mathrm{T}}$ : Retention time.

\section{REFERENCES}

1. Cawley AT, Trout GJ, Kazlauskas R, Howe, CJ, George AV. Carbon isotope ratio (delta13C) values of urinary steroids for doping control in sport. Steoids. 2009;74(3):375-79.

2. The World Anti-Doping Agency (WADA). Prohibited List. 2019.

3. Chung BC, Choo HY, Kim TW, Eom KD, Kwon OS, Suh J, et al. Analysis of anabolic steroids using GC/MS with selected ion monitoring. J Anal Toxicol. 1990;14(2):91-5.

4. Coppen JJW. Steroids from plants to pills-The changing the picture. Trop Sci. 1979;21:125-41.

5. Ueki M, Okano M. Analysis of exogenous dehydroepiandrosterone excretion in urine by gas chromatography/combustion/isotope ratio mass spectrometry. Rapid Commun Mass Spectrom. 1999;13(22):2237-43.

6. Becchi M, Aguilera R, Farizon, Y, Flament AA, Casabianca H, James P. Gas chromatography/combustion/isotope-ratio mass spectrometry analysis of urinary steroids to detect misuse of testosterone in sport. Rapid Commun Mass Spectrom. 1994;8(4):304-8.

7. Aguilera R, Becchi M, Casabianca H, Hatton CK, Catlin DH, Starcevic B, et al. Improved method of detection of testosterone abuse by gas chromatography/ combustion/isotope ratio mass spectrometry analysis of urinary steroids. Mass Spectrom. 1996;31(2):169-76.

8. Shackleton CHL, Roitman, E, Phillips A, Chang TY. Androstanediol and 5-androstenediol profiling for detecting exogenously administered dihydrotestosterone, epitestosterone and dehydroepiandrosterone: Potential use in gas chromatography isotope ratio mass spectrometry. Steoids. 1997;62(10):379-85.

9. Cawley AT, Flenker UJ. The application of carbon isotope ratio mass spectrometry to doping control. Mass Spectrom. 2008;43(7):854-58.

10. Mareck U, Geyer H, Opfermann, G, Thevis M, Schanzer WJ. Factors influencing the steroid profile in doping control analysis. Mass Spectrom. 2008;43(7):877-91.

11. Shackleton $\mathrm{CHL}$, Roitman E, Phillips A, Chang T. Androstanediol and 5-androstenediol profiling for detecting exogenously administered dihydrotestosterone, epitestosterone and dehydroepiandrosterone: Potential use in gas chromatography isotope ratio mass spectrometry. Steoids. 1997;62(10):665-73. 
12. Aguilera R, Catlin DH, Becchi M, Phillips AW, Swerdloff, RS, Pope HG. Hatton CK. Screening urine for exogenous testosterone by isotope ratio mass spectrometric analysis of one pregnanediol and two androstanediols, J Chromatogr B. 1999;727(1-2):95-105.

13. Aguilera R, Chapman TE, Catlin DH. Performance Characteristics of a Carbon Isotope Ratio Method for Detecting Doping with Testosterone Based on Urine Diols: Controls and Athletes with Elevated Testosterone/Epitestosterone Ratios, drug monitoring and toxicology. Clin Chem. 2001;47(2):292-300.

14. Kang ML, Ho JK, Eun SJ, Hye HY, Oh SK, Changbae J, et al. Validation and Applications of Gas Chromatography-Combustion/isotope
Ratio Mass Spectrometric Method to Control Misuse of Androgens in Human Mass Spectrom Lett. 2011;2(2):33-6.

15. Jain S, Lal R, Garg T, Haseen J, Goswami M, Vandana, et al. Mareck U. (eds.) Recent Advances in Doping Analysis, Köln. 2010

16. Piper T, Mareck U, Geyer H, Flenker U, Thevis M, Platen P, et al Determination of $13 \mathrm{C} / 12 \mathrm{C}$ ratios of endogenous urinary steroids: Method validation, reference population and application to doping control purposes. Rapid Commun Mass Spectrom. 2008;22(14):2161-5.

17. Saudan C, Emery C, Marclay F, Strajm E, Mangin P, Saugy MJ. Validation and performance comparison of two carbon isotope ratio methods to control the misuse of androgens in humans. Chromatogr B. 2009;877(23):2321-25.

\section{PICTORIAL ABSTRACT}

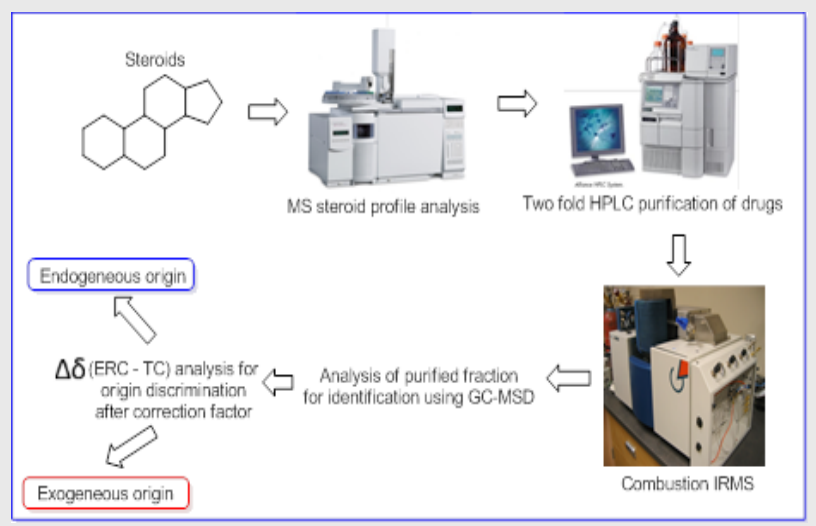

\section{SUMMARY}

The present review describes an improved method for the discrimination of endogenous and exogenous origin of steroids and the same was also found suitable for confirmation analysis. The sample preparation and purification procedure was employed successfully in sport events of international fame and various athletes were caught using endogenous steroids. The method successfully cleared various proficiency testing rounds by WADA, WAADS and other proficiency testing agencies and thus found fit for purpose.

Cite this article: Shrivastava A, Jain S, Sahu PL, Shukla S, Sahu K. Improved Method for Differentiation of Synthetic and Natural Endogenous Anabolic Steroids using Gas Chromatography Isotope Ratio Mass Spectrometry (GC/C/ IRMS) Followed by Two-Fold High Performance Liquid Chromatography (HPLC) Cleanup Method: A Perspective. Indian J of Pharmaceutical Education and Research. 2019;53(4s):s460-s465. 